\title{
THE SURPRISING ABSENCE OF ABSORPTION IN THE FAR-ULTRAVIOLET SPECTRUM OF Mrk 231
}

\author{
S. Veilleux ${ }^{1,2,3,4}$, M. Trippe ${ }^{1}$, F. HamanN ${ }^{5}$, D. S. N. Rupke ${ }^{6}$, T. M. Tripp ${ }^{7}$, H. Netzer ${ }^{8}$, D. Lutz ${ }^{4}$, K. R. Sembach ${ }^{9}$, H. Krug $^{1}$, \\ S. H. Teng ${ }^{10}$, R. Genzel ${ }^{4}$, R. Maiolino ${ }^{11,12}$, E. Sturm ${ }^{4}$, And L. TACCOni ${ }^{4}$ \\ ${ }^{1}$ Department of Astronomy, University of Maryland, College Park, MD 20742, USA; hkrug@astro.umd.edu, veilleux@astro.umd.edu, trippe@astro.umd.edu \\ 2 Joint Space-Science Institute, University of Maryland, College Park, MD 20742, USA \\ ${ }^{3}$ Astroparticle Physics Laboratory, NASA Goddard Space Flight Center, Greenbelt, MD 20771, USA \\ ${ }^{4}$ Max-Planck-Institut für extraterrestrische Physik, Postfach 1312, D-85741 Garching, Germany \\ ${ }^{5}$ Department of Astronomy, University of Florida, Gainesville, FL 32611, USA \\ ${ }^{6}$ Department of Physics, Rhodes College, Memphis, TN 38112, USA \\ ${ }^{7}$ Department of Astronomy, University of Massachussetts, Amherst, MA 01003, USA \\ ${ }^{8}$ School of Physics and Astronomy and the Wise Observatory, The Raymond and Beverly Sackler Faculty of Exact Sciences, \\ Tel-Aviv University, Tel-Aviv 69978, Israel \\ ${ }^{9}$ Space Telescope Science Institute, Baltimore, MD 21218, USA \\ ${ }^{10}$ Observational Cosmology Laboratory, NASA Goddard Space Flight Center, Greenbelt, MD 20771, USA \\ ${ }^{11}$ Cavendish Laboratory, University of Cambridge, 19 J. J. Thomson Avenue, Cambridge CB3 OHE, UK \\ ${ }^{12}$ Kavli Institute for Cosmology, Madingley Road, Cambridge CB3 OHA, UK \\ Received 2012 August 24; accepted 2012 December 10; published 2013 January 21
}

\begin{abstract}
Mrk 231, the nearest $(z=0.0422)$ quasar, hosts both a galactic-scale wind and a nuclear-scale iron low-ionization broad absorption line (FeLoBAL) outflow. We recently obtained a far-ultraviolet (FUV) spectrum of this object covering 1150-1470 ̊ with the Cosmic Origins Spectrograph on board the Hubble Space Telescope. This spectrum is highly peculiar, highlighted by the presence of faint ( $\lesssim 2 \%$ of predictions based on $\mathrm{H} \alpha$ ), broad $\left(\gtrsim 10,000 \mathrm{~km} \mathrm{~s}^{-1}\right.$ at the base), and highly blueshifted (centroid at $\sim-3500 \mathrm{~km} \mathrm{~s}^{-1}$ ) Ly $\alpha$ emission. The FUV continuum emission is slightly declining at shorter wavelengths (consistent with $F_{\lambda} \propto \lambda^{1.7}$ ) and does not show the presence of any obvious photospheric or wind stellar features. Surprisingly, the FUV spectrum also does not show any unambiguous broad absorption features. It thus appears to be dominated by the AGN, rather than hot stars, and virtually unfiltered by the dusty FeLoBAL screen. The observed Ly $\alpha$ emission is best explained if it is produced in the outflowing BAL cloud system, while the Balmer lines arise primarily from the standard broad emission line region seen through the dusty $\left(A_{V} \sim 7 \mathrm{mag}\right.$ ) broad absorption line region. Two possible geometric models are discussed in the context of these new results.
\end{abstract}

Key words: galaxies: active - ISM: jets and outflows - line: formation - quasars: absorption lines - quasars: individual (Mrk 231)

Online-only material: color figures

\section{INTRODUCTION}

At a distance ${ }^{13}$ of only $178 \mathrm{Mpc}$, where $1^{\prime \prime}=0.863 \mathrm{kpc}$, Mrk 231 is the nearest quasar known (Boksenberg et al. 1977). The analysis by Braito et al. (2004) of the joint XMMNewton-BeppoSAX X-ray spectrum of this object has revealed the existence of a highly absorbed $\left(N_{\mathrm{H}} \sim 2 \times 10^{24} \mathrm{~cm}^{-2}\right)$, powerful (intrinsic 2-10 keV luminosity of $\sim 1 \times 10^{44} \mathrm{erg} \mathrm{s}^{-1}$ ) AGN. Mrk 231 is also an ultraluminous infrared galaxy (ULIRG) with infrared $(8-1000 \mu \mathrm{m})$ luminosity $\log \left[L_{\mathrm{IR}} / L_{\odot}\right]=12.54$ and a bolometric luminosity $\log \left[L_{\mathrm{BOL}} / L_{\odot}\right] \approx 12.60 .{ }^{14} \mathrm{~A}$ detailed analysis of its Spitzer mid-infrared spectrum indicates that $\sim 30 \%$ of this bolometric luminosity is produced by a circumnuclear starburst with SFR $\sim 170 M_{\odot} \mathrm{yr}^{-1}$, surrounding the dominant optical broad-lined quasar (Veilleux et al. 2009). The disturbed morphology of the host suggests that both the quasar and starburst are triggered by a recent merger event (e.g., Hamilton \& Keel 1987; Hutchings \& Neff 1987; Surace et al. 1998; Veilleux et al. 2002, 2006).

\footnotetext{
13 Based on a redshift $z=0.0422$ and a cosmology with $H_{0}=73 \mathrm{~km} \mathrm{~s}^{-1}$ $\mathrm{Mpc}^{-1}, \Omega_{\text {matter }}=0.27$, and $\Omega_{\text {vacuum }}=0.73$.

14 This estimate for the bolometric luminosity includes all IR and non-IR contributions and is derived by simply assuming $L_{\mathrm{BOL}}=1.15 \mathrm{~L}_{\mathrm{IR}}$, typical for ULIRGs (e.g., Sanders \& Mirabel 1996).
}

In recent years, Mrk 231 has become the archetype of galacticscale quasar-driven winds. These outflow events are purported to self-regulate the growth of the black hole $(\mathrm{BH})$ and the spheroidal component of the galaxy, and explain the relatively tight BH-spheroid-mass relation (e.g., Di Matteo et al. 2005; Murray et al. 2005; Veilleux et al. 2005 and references therein). Evidence in Mrk 231 for a powerful kpc-scale wind with outflow velocity of up to $\sim 1000 \mathrm{~km} \mathrm{~s}^{-1}$ and mass outflow rate of the order of $\sim 400-1000 M_{\odot} \mathrm{yr}^{-1}$ has now been seen in the neutral gas (Rupke et al. 2005; Rupke \& Veilleux 2011, 2012), the molecular gas (Feruglio et al. 2010; Fischer et al. 2010; Sturm et al. 2011; Aalto et al. 2012; Cicone et al. 2012), and the warm ionized gas (Lipari et al. 2009; Rupke \& Veilleux 2011, 2012). It is not clear whether this galactic-scale wind is physically related to the well-known unresolved broad absorption-line (BAL) systems in the core of this object (Boksenberg et al. 1977; Rudy et al. 1985; Hutchings \& Neff 1987; Boroson et al. 1991; Kollatschny et al. 1992; Forster et al. 1995; Smith et al. 1995; Rupke et al. 2002; Gallagher et al. 2002, 2005). The Mrk 231 BAL features are detected in several transitions including many low-ionization species (e.g., Na I D $\lambda \lambda 5890$, 5896 , $\mathrm{He}^{*} \lambda 3889$, Ca II $\mathrm{H}$ and $\mathrm{K}$, and $\mathrm{Mg}$ II $\lambda 2800$, as well as Fe II UV1 $\lambda 2599$ and Fe II UV2 $\lambda 2382$, hence fitting the rare FeLoBAL category). They show strong absorption across the 
range $\sim-3500$ to $-5500 \mathrm{~km} \mathrm{~s}^{-1}$ and no significant absorption at velocities above $-3500 \mathrm{~km} \mathrm{~s}^{-1}$ (see, e.g., Rupke et al. 2002).

Mrk 231 was the first target observed under our farultraviolet (FUV) spectroscopic survey of the nearby QUEST (Quasar-ULIRG Evolution Study; e.g., Veilleux 2012 and references therein) QSOs with the Hubble Space Telescope (HST; PID 12569, PI: Veilleux). The unprecedented sensitivity of the Cosmic Origins Spectrograph (COS; Green et al. 2012) allowed us to obtain the first high signal-to-noise $(\mathrm{S} / \mathrm{N})$ ratio spectrum of Mrk 231 below $\sim 1500 \AA$. This paper describes the results from our analysis of this spectrum. The results from the survey will be presented in future papers. The acquisition and reduction of the COS data and complementary optical spectrum of Mrk 231 are discussed in Section 2, followed by a description of the results (Section 3 ) and a discussion of the implications (Section 4). Throughout this paper, we use $z=0.0422$ as the rest-frame velocity of Mrk 231; this value is considered quite secure since it is based on rotation curves derived from the ionized, neutral, and molecular gas components and the peak of the H I $21 \mathrm{~cm}$ absorption feature (see Rupke \& Veilleux 2011 for references).

\section{OBSERVATIONS}

\subsection{HST-COS FUV Spectroscopy}

Mrk 231 was observed for a total 12,539 s spread over five orbits using the G130M grating and the $2 . .5 \times 2$ ".5 aperture to cover $\sim 1150-1470 \AA$ at a spectral resolution of $\sim 15-20 \mathrm{~km} \mathrm{~s}^{-1}$. All of these observations were done in TIME-TAG mode to provide temporal sampling and allow us to exclude poor quality data and get improved thermal correction and background removal. In the end, we did not have to exclude any data for Mrk 231. To reduce the fixed pattern noise and fill up the wavelength hole produced by the chip gap without excessive overheads, we split each orbit into four separate segments of similar durations at two different FP_POS offset positions (either segments 1 and 3 or segments 2 and 4, depending on the orbit) and two different central wavelengths (1309 or $1327 \AA$ ). All observations were carried out on 2011 October 15 and processed using the standard $\operatorname{calcos}^{15}$ calibration pipeline. Flat fielding, alignment, and co-addition of the individual exposures were performed using the IDL routines provided by the COS GTO team. $^{16}$

\subsection{Ground-based Optical Spectroscopy}

The highest-velocity $\left(\sim-8000 \mathrm{~km} \mathrm{~s}^{-1}\right)$ component of the BAL systems in Mrk 231 has proven to be time variable (Hutchings \& Neff 1987; Boroson et al. 1991; Kollatschny et al. 1992; Forster et al. 1995; Rupke et al. 2002, 2005), so we decided to get a new optical spectrum of this object to complement our 2001, 2004, and 2007 spectra published in Rupke et al. (2002), Rupke et al. (2005c), and Rupke \& Veilleux (2011), respectively, and facilitate the task of comparing these data with the COS FUV spectrum. The instrumental setup used for these ground-based observations was the same as that used for the 2004 optical spectra: the Ritchey-Chrétien spectrograph on the Mayall 4 m telescope at Kitt Peak was used on 2012 April 27

\footnotetext{
15 See Chapter 3 of Part II of the HST Data Handbook for COS (http://www.stsci.edu/hst/cos/documents/handbooks/datahandbook) for the details of the calcos processing.

16 Routines are available at

http://casa.colorado.edu/ danforth/science/cos/costools.html, and described in Danforth et al. (2010) and Keeney et al. (2012).
}

with grating KPC-18C in the first order and a 1".25 slit to obtain 4 spectra each of 15 minute duration with a spectral resolution of $85 \mathrm{~km} \mathrm{~s}^{-1}$. Standard IRAF $^{17}$ procedures were used to reduce and calibrate this spectrum.

\section{RESULTS}

A near-ultraviolet (NUV) COS image of Mrk 231, obtained for accurate target acquisition, is consistent with an unresolved point source centered on the optical position of the quasar at the resolution of the COS imager (FWHM $\sim 0$ '.05). The FUV spectrum of Mrk 231 is shown in Figures 1 and 2 and is highly peculiar. We have been able to identify unambiguously only one emission feature intrinsic to Mrk 231: broad, highly blueshifted Ly $\alpha$ emission (discussed in detail in the last paragraph of this section). This lack of FUV features contrasts starkly with the rich optical spectrum of this object (shown in Figure 3) and with the rest-frame UV spectra of other FeLoBAL quasars (e.g., Hall et al. 2002). These and other quasar spectra indicate that Mrk 231 should have strong broad absorption in Ly $\alpha$ and possibly also emission and absorption from $\mathrm{P} v \lambda \lambda 1118$, 1128, Si III $\lambda 1206$, N v $\lambda \lambda 1238,1243$, Si II $\lambda 1260$ (Si II* $\lambda 1265)$, O I $\lambda 1302$, Si II $\lambda 1304$ (Si II* $\lambda 1309)$, C II $\lambda 1335$ (C II* $\lambda 1336)$, and Si IV $\lambda \lambda 1394,1403$ within our spectral coverage. However, none of these features is clearly detected. The peculiarity of the UV spectrum of Mrk 231 was first noted by Hutchings \& Neff (1987) and Lipari et al. (1994) based on IUE spectra. It was further investigated by Smith et al. (1995) using NUV FOS spectropolarimetric data and by Gallagher et al. (2002) using a short FOS calibration observation in the archives. In particular, Gallagher et al. (2002) noted the likely presence of broad C IV $\lambda \lambda 1548,1550$ emission affected by a weak BAL, but the $\mathrm{S} / \mathrm{N}$ ratio of the FOS data was insufficient to provide firm limits on the equivalent widths of the emission and absorption features below $\sim 1450 \AA$. The new COS data show that the spectrum of Mrk 231 at $<1450 \AA$ is also highly unusual. As discussed in Section 4.3, we believe that this is the result of a complex geometry with a dusty broad absorption line region (BALR) obscuring portions of both the continuum source and standard broad emission line region (BELR).

While the COS spectrum of Mrk 231 is dominated by Ly $\alpha$ emission, it does also present a number of faint but significant "bumps" and "wiggles." We are confident that these features are real since they are seen in individual exposures with different FP_POS and central wavelengths during each of the five orbits, but they are not seen in the FUV spectra of our other targets, which were observed in the same fashion reaching similar $\mathrm{S} / \mathrm{N}$ ratios. Table 1 lists the properties of these weak features along with tentative identifications (IDs). None of these IDs is secure except for the narrow absorption feature at $1252 \AA$, which is most likely a weak narrow $\mathrm{HI}$ absorber at $\sim-3530 \mathrm{~km} \mathrm{~s}^{-1}$ associated with either the nuclear outflow in Mrk 231 or IGM along our line of sight. The significant width $\left(\sim 2000 \mathrm{~km} \mathrm{~s}^{-1}\right)$ of the absorption feature at $\sim 1392 \AA$ suggests an AGN origin. If this feature forms in the $\sim-4500 \mathrm{~km} \mathrm{~s}^{-1}$ BAL outflow seen in the optical $\mathrm{Na}$ I, $\mathrm{Ca}$ II, and $\mathrm{He}$ I lines, then the rest wavelength of the $1392 \AA$ feature should be $\sim 1356 \AA$, for which there is no plausible ID. The strongest absorption line we expect to find near these wavelengths is $\mathrm{C}$ II $\lambda 1335$ (+ $\left.\mathrm{CII}^{*} \lambda 1336\right)$, but this implies much lower velocities, only $+200(-70) \mathrm{km} \mathrm{s}^{-1}$, compared to the optical BALs. Nonetheless, this ID for the $1392 \AA$ feature is consistent with the weaker but similar-looking absorption dip at

\footnotetext{
17 http://iraf.noao.edu/
} 


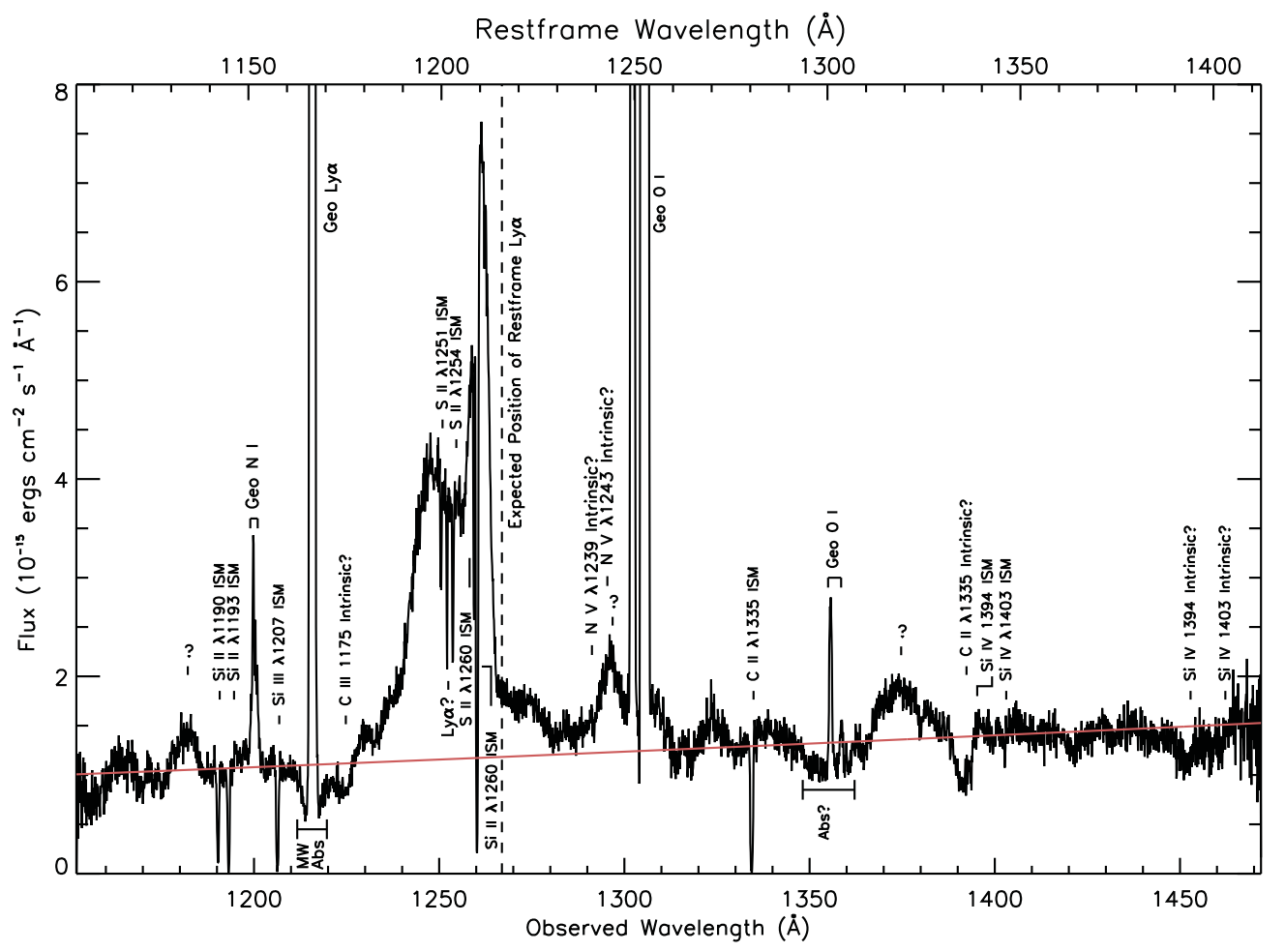

Figure 1. HST-COS FUV spectrum of Mrk 231, binned by 10 spectral pixels to help show the fainter features. It has not been corrected for the small foreground Galactic extinction $\left(A_{V} \sim 0.03 \mathrm{mag}\right)$. This spectrum is dominated by broad, highly blueshifted Ly $\alpha$ emission. The FUV continuum emission is nearly featureless and only slowly declining at shorter wavelengths, consistent with $F_{\lambda} \propto \lambda^{1.7}$ (shown as a red line in the figure). It is thus dominated by the AGN and only slightly affected by dust reddening $\left(A_{V} \sim 0.5 \mathrm{mag}\right.$.) from the neutral and dusty broad absorption line region.

(A color version of this figure is available in the online journal.)

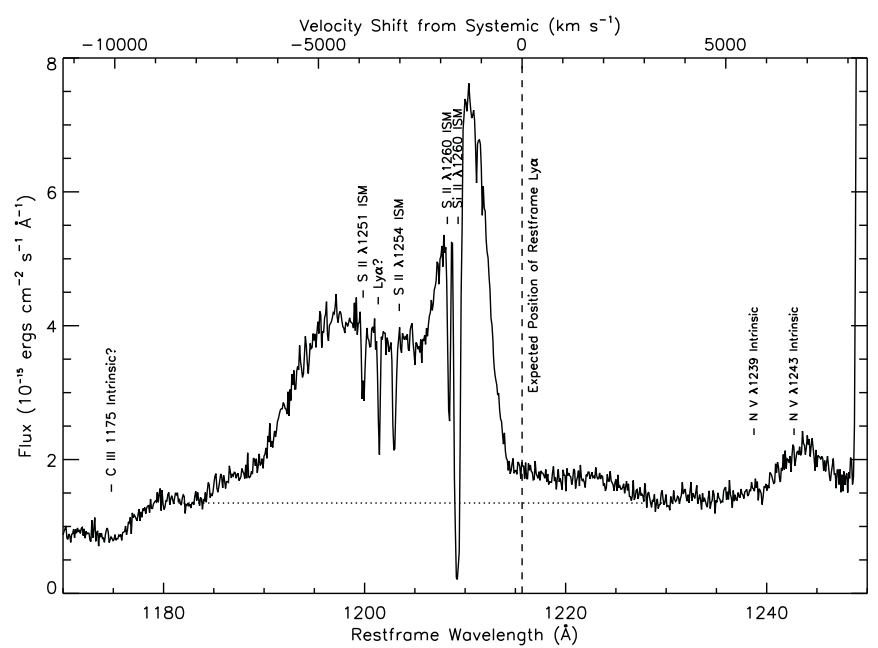

Figure 2. Ly $\alpha$ emission profile in Mrk 231. The vertical dashed line indicates the expected position of rest-frame Ly $\alpha$, while the dotted line indicates a conservatively high estimate of the continuum level used to produce Figure 4. Note the large blueshift of the $\mathrm{Ly} \alpha$ emission with respect to systemic (the centroid of the profile is at $\sim-3500 \mathrm{~km} \mathrm{~s}^{-1}$ ) and the width of the line $\left(\gtrsim 10,000 \mathrm{~km} \mathrm{~s}^{-1}\right.$ at the base of the profile) which indicates an AGN origin. There is no indication that Si II $\lambda \lambda 1190,1193$, N I $\lambda 1200$, and Si III $\lambda 1207$ are significantly affecting the profile of $\operatorname{Ly} \alpha$. The feature at restframe wavelength $1243.5 \AA$ is unidentified, but it could plausibly be highly redshifted Ly $\alpha$ emission at $\sim+6900 \mathrm{~km} \mathrm{~s}^{-1}$ (see Section 3 and Table 1 for more detail).

$\sim 1452 \AA$, which might be Si IV $\lambda 1394$ at a similar near-systemic velocity. The apparent absence of absorption in the other half of the Si Iv doublet, at $1403 \AA$, is consistent with its factor of two lower $f$-value and the lower $\mathrm{S} / \mathrm{N}$ ratio in this part of the measured spectrum. The power-law fit in Figure 1 also suggests the presence of shallow absorption features around $\sim 1355 \AA$

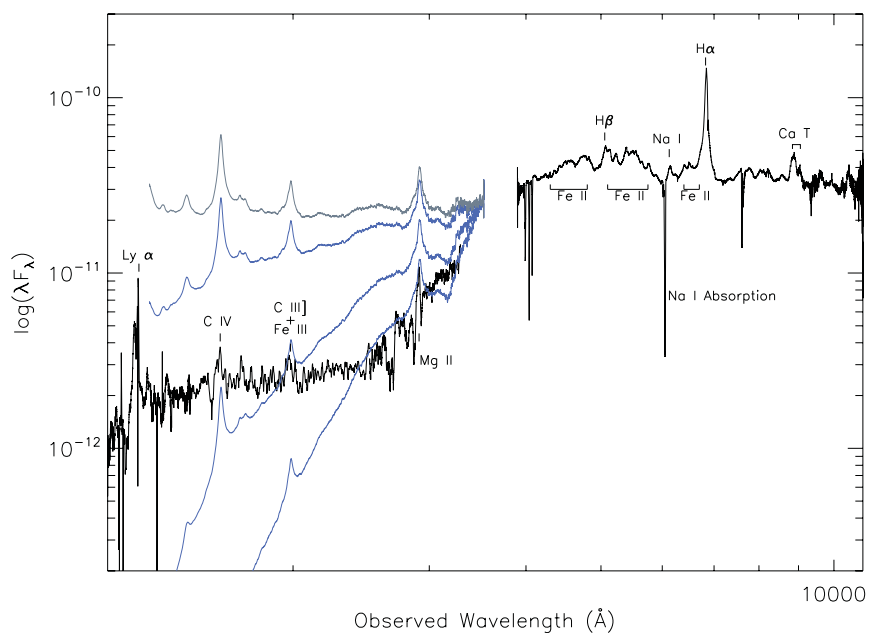

Figure 3. UV-optical spectral energy distribution (SED) of Mrk 231 in $\log \lambda f_{\lambda}$ vs. $\log \lambda$ (observed) units. This SED combines the new COS data with archival FOS spectra and the 2001 Keck/ESI optical spectrum published in Rupke et al. (2002). The old pre-COSTAR FOS NUV spectrum at 2500-3300 $\AA$ was scaled up to match the data at shorter wavelengths (see Section 4.1 for more detail). A comparison with our new KPNO spectrum shows no evidence for variations in the optical continuum and line emission of Mrk 231. The KPNO spectrum is not shown here because it does not cover as broad a wavelength range as the Keck data. The blue curves indicate the $H S T / F O S$ composite quasar spectrum used in the HST COS ETC (shown in gray) reddened by $A_{V}=1.0,4.0$, and 7.0 mag using $R_{V}=A_{V} / E(B-V)=3.1$ and an extinction curve without the $2175 \AA$ dust absorption feature (Conroy et al. 2010), normalized to match the observed spectrum of Mrk 231 at $3600 \AA$. Note the dramatic drop in flux in Mrk 231 from $\sim 4000 \AA$ to $\sim 2500 \AA$, requiring dust reddening $A_{V} \sim 7 \mathrm{mag}$, followed by a slowly declining continuum below that wavelength, only slightly affected by dust $\left(A_{V} \sim 0.5 \mathrm{mag}\right)$. This is consistent with the near-zero polarization at $\sim 1800$ Å found by Smith et al. (1995).

(A color version of this figure is available in the online journal.) 
Table 1

Uncertain Features in the COS Spectrum

\begin{tabular}{|c|c|c|c|c|c|c|}
\hline $\begin{array}{l}\lambda_{\text {obs }} \\
(\AA) \\
(1)\end{array}$ & $\begin{array}{l}\lambda_{\text {rest }} \\
(\AA) \\
(2)\end{array}$ & $\begin{array}{l}\text { EW } \\
(\AA) \\
(3)\end{array}$ & $\begin{array}{c}F_{\mathrm{em}} \\
\left(10^{-15} \mathrm{erg} \mathrm{s}^{-1} \mathrm{~cm}^{-2}\right)\end{array}$ & $\begin{array}{l}\text { Possible IDs } \\
\text { (5) }\end{array}$ & $\begin{array}{c}\text { Velocity } \\
\left(\mathrm{km} \mathrm{s}^{-1}\right) \\
(6)\end{array}$ & $\begin{array}{c}\text { Confidence } \\
\text { (7) }\end{array}$ \\
\hline \multirow[t]{2}{*}{$1181.3(0.8)$} & 1133.5 & $-3.3(0.3)$ & $3.6(0.8)$ & $\mathrm{N}_{\mathrm{I}} \lambda 1134.4$ & -240 & 3 \\
\hline & & & & Fe II $\lambda 1144.0$ & -2750 & 3 \\
\hline $1225.4(0.1)$ & 1175.8 & $+0.8(0.2)$ & $\cdots$ & C III $\lambda 1175.6$ & +50 & 3 \\
\hline $1252.0(0.4)$ & 1201.3 & $-29.2^{\mathrm{a}} /-46.6^{\mathrm{a}}$ & $49^{\mathrm{a}} / 85^{\mathrm{a}}$ & Ly $\alpha \lambda 1215.7$ & -3550 & 0 \\
\hline $1252.1(0.05)$ & 1201.4 & $+0.13(0.02)$ & $\cdots$ & Ly $\alpha \lambda 1215.7$ & -3530 & 1 \\
\hline \multirow[t]{3}{*}{$1296.0(0.3)$} & 1243.5 & $-3.6(0.8)$ & $6.4(1.4)$ & Ly $\alpha \lambda 1215.7$ & +6900 & 3 \\
\hline & & & & N v $\lambda 1242.8$ & +170 & 3 \\
\hline & & & & Si II $\lambda 1260.4$ UV4 & -4020 & 3 \\
\hline $1324.0(0.2)$ & 1270.4 & $-2.6(0.5)$ & $2.7(0.5)$ & $? ? ?$ & $? ? ?$ & 3 \\
\hline $1339.5(0.5)$ & 1285.2 & $-4.1(1.2)$ & $4.7(0.9)$ & O I $\lambda 1302.2+$ Si II $\lambda 1304.4$ & -4200 & 3 \\
\hline $1355.2(0.1)$ & 1300.3 & $+2.7(0.6)$ & $\cdots$ & O I $\lambda 1302.2+$ Si II $\lambda 1304.4$ & -740 & 3 \\
\hline \multirow[t]{3}{*}{$1375.0(0.7)$} & 1319.3 & $-8.2(2.0)$ & $13.0(1.7)$ & Ni II $\lambda 1317.2$ & +480 & 3 \\
\hline & & & & C I $\lambda 1328.8$ & -2140 & 3 \\
\hline & & & & C II $\lambda 1334.5$ UV1 & -3410 & 3 \\
\hline \multirow[t]{2}{*}{$1391.8(0.1)$} & 1335.4 & $+1.8(0.2)$ & $\cdots$ & $\mathrm{C}_{\text {II }} \lambda 1334.5$ UV1 & +200 & 2 \\
\hline & & & $\cdots$ & C II* $\lambda 1335.7$ UV1 & -70 & 2 \\
\hline $1452.0(0.4)$ & 1393.2 & $+0.7(0.1)$ & $\cdots$ & Si IV $\lambda 1393.8$ & -130 & 2 \\
\hline $1462.0(1.0)$ & 1402.8 & $+0.2(0.2)$ & $\cdots$ & Si IV $\lambda 1402.8$ & -0 & 2 \\
\hline
\end{tabular}

Notes. Column 1: wavelength of the feature (centroid) in the observer's frame in $\AA$. Column 2 : wavelength of the feature (centroid) in the rest frame $(z=$ 0.0422) in $\AA$. Column 3: equivalent width in $\AA$ measured in the observer's frame (negative value corresponds to emission). Column 4: flux of the emission feature in $10^{-15} \mathrm{erg} \mathrm{s}^{-1} \mathrm{~cm}^{-2}$. Column 5: possible line identification based on, e.g., Morton (2003), Laor et al. (1997), Zheng et al. (1997), vanden Berk et al. (2001), and Hall et al. (2002). Column 6: velocity of the feature (centroid) corresponding to the tentative line identification. Column 7: level of confidence of the line identification: $0=$ certain, $1=$ confident, $2=$ uncertain, $3=$ unlikely. See Section 3 for more detail.

a The first number corresponds to the core of the Ly $\alpha$ emission line, while the second number also includes the contribution from the broad wings.

$(\sim 1300 \AA$ in the rest frame) and $\sim 1225 \AA(\sim 1176 \AA$; partly affected by the damping wings of Galactic Ly $\alpha$ absorption). This latter feature may be stellar photospheric $\mathrm{C}_{\text {III }} \lambda 1176$ near systemic velocity; we return to this identification in Section 4.1.

The three emission features at $\sim 1181,1296$, and $1375 \AA$ could not be matched unambiguously to the redshifted atomic transitions expected from Mrk 231 (or the "template" narrowline Seyfert 1; e.g., Laor et al. 1997). The feature at $1181 \AA$ may be N I $\lambda 1134$ blueshifted by $\sim-200 \mathrm{~km} \mathrm{~s}^{-1}$ with respect to the systemic velocity of Mrk 231, but this identification is doubtful since the expected stronger $\mathrm{N}$ I $\lambda 1200$ transition is not clearly detected (although blending with the highly blueshifted Ly $\alpha$ emission could hide some $\mathrm{N}_{\mathrm{I}} \lambda 1200$ emission). Another possibility is Fe II $\lambda 1144$ blueshifted by $-2750 \mathrm{~km} \mathrm{~s}^{-1}$. The broad feature at $1375 \AA$ could plausibly be a blend of Ni II $\lambda 1317$ and $\mathrm{CI}$ $\lambda 1329$, but with peculiar shifts of about +500 and $-2200 \mathrm{~km} \mathrm{~s}^{-1}$ with respect to systemic, respectively. We consider this identification unlikely because (1) these lines are not seen in I Zw 1 and (2) given the ionization potentials and abundances of $\mathrm{Ni}$ and $\mathrm{C}$, many other lines should also be present in the COS spectrum if those IDs were correct, but they are not detected. Highly blueshifted ( $\left.-3410 \mathrm{~km} \mathrm{~s}^{-1}\right) \mathrm{C}_{\text {II }} \lambda 1334.5$ is perhaps a more likely possibility. The $1296 \AA$ feature could conceivably be residual $\mathrm{N} v \lambda 1243$ ( + highly redshifted $\mathrm{Ly} \alpha$ ) emission affected by strong $\mathrm{Nv}$ BAL, but the $\mathrm{Nv}+\mathrm{Ly} \alpha+$ continuum emission over 1270-1290 Å would have to conspire with the N v BAL to produce a nearly featureless pseudo-continuum and explain the absence of the Nv $\lambda 1238$ doublet line, which seems very unlikely. Highly blueshifted (-4000 $\mathrm{km} \mathrm{s}^{-1}$ ) Si II $\lambda 1260$ may also contribute to this feature. Similarly, the weaker feature near $1339 \AA$ may be a blend of O I $\lambda 1302$ and Si II $\lambda 1304$ blueshifted by $-4500 \mathrm{~km} \mathrm{~s}^{-1}$. These large blueshifts would be unusual for quasars, but as we discuss below, they would be similar to the shift measured in the Ly $\alpha$ emission feature. A similar blueshift $\left(-3000 \mathrm{~km} \mathrm{~s}^{-1}\right)$ has been reported in C III $\lambda 1909$, but this feature is badly blended with Fe III lines at shorter wavelengths (Smith et al. 1995; Gallagher et al. 2002). The C IV emission feature in the data of Gallagher et al. (2002) also seems blueshifted, but the blueshifted C IV absorption feature complicates the analysis. We return to this point in Section 4.3.

The Ly $\alpha$ emission feature of Mrk 231 is very unusual (Figure 2). It is centered at $\sim 1252 \AA$ or $1201 \AA$ in the restframe, i.e., blueshifted by $\sim 3500 \mathrm{~km} \mathrm{~s}^{-1}$ with respect to rest (assuming no contribution from Si II $\lambda \lambda 1190,1193$, N I $\lambda 1200$, and Si III $\lambda 1207$ emission). It has a very peculiar profile with a narrow peak centered at $\sim 1261 \AA\left(\sim-1500 \mathrm{~km} \mathrm{~s}^{-1}\right.$ in the rest frame) and wings extending over at least $1240-1265 \AA$ $\left(\sim 6000 \mathrm{~km} \mathrm{~s}^{-1}\right)$ and quite possibly as much as $1225-1280 \AA$ $\left(\sim 13,000 \mathrm{~km} \mathrm{~s}^{-1}\right)$. The broad width clearly points to an AGN origin for this feature. The Ly $\alpha$ emission is centered on the NUV nucleus and spatially unresolved with the rather coarse spatial resolution of COS at $1250 \AA$ for our settings $\left(\sim 1^{\prime \prime} .3\right.$; Ghavamian et al. 2010). The integrated Ly $\alpha$ flux is $\sim 4.9 \times$ $10^{-14} \mathrm{erg} \mathrm{s}^{-1} \mathrm{~cm}^{-2}$ in the line core and $8.5 \times 10^{-14}$ if one includes the broad wings (with corresponding rest equivalent widths of 29.2 and $46.6 \AA$, respectively; see Table 1). In comparison, the $\mathrm{H} \alpha$ flux measured from our $2012 \mathrm{KPNO}$ spectrum is $\sim 140 \times 10^{-14} \mathrm{erg} \mathrm{s}^{-1} \mathrm{~cm}^{-2}$ (the same within the uncertainties as that measured from our older ESI spectrum). The $\mathrm{Ly} \alpha / \mathrm{H} \alpha$ flux ratio of Mrk 231 is therefore only $\sim 0.5 \%$ of the high-density case B value of about 13 . Collisional suppression of the level 2 population in hydrogen is known to be important in the high-density BELRs of quasars but reduces the $\mathrm{Ly} \alpha / \mathrm{H} \alpha$ ratio by at most a factor of $\sim 2-3$ relative to the Case B value (e.g., Netzer et al. 1995). The integrated $\mathrm{Ly} \alpha / \mathrm{H} \alpha$ ratio in Mrk 231 is thus $\lesssim 2 \%$ the values typically measured in BLRs. This already small ratio is further reduced 


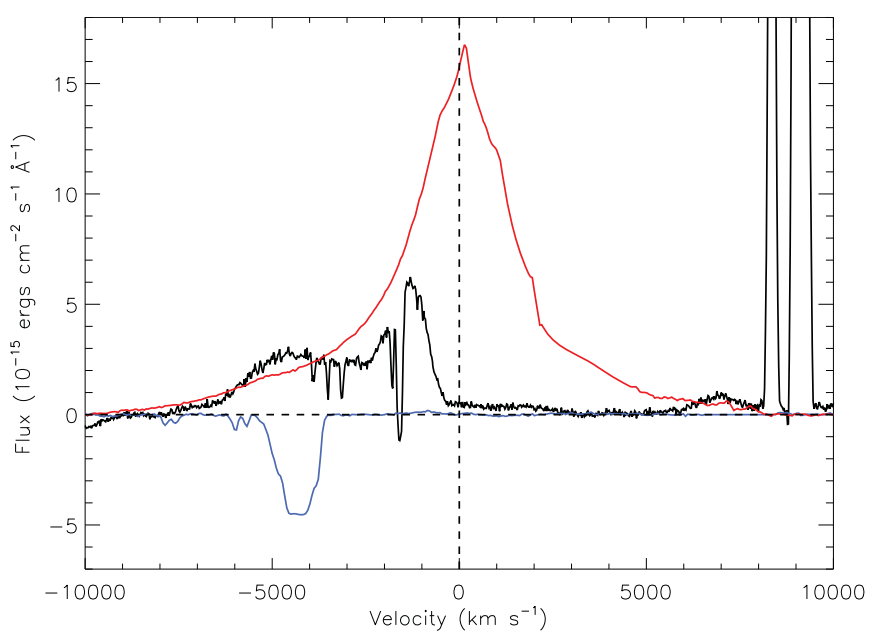

Figure 4. Comparison of the continuum-subtracted $\mathrm{H} \alpha$ emission (red), Ly $\alpha$ emission (black), and Na I D absorption (blue). The conservatively high continuum level shown in Figure 2 was used to derive the Ly $\alpha$ emission profile shown here. The optical features are derived from our most recent KPNO data to reduce profile variability. All three features are on the same velocity and absolute flux scales. The $\mathrm{H} \alpha$ profile was interpolated at $\sim 1000-2000 \mathrm{~km} \mathrm{~s}^{-1}$ to remove the telluric $\mathrm{O}_{2} \lambda 6850$ band. Ly $\alpha$ shares a stronger resemblance with $\mathrm{Na}$ $\mathrm{D}$ absorption than with $\mathrm{H} \alpha$ emission. The emission feature at $\sim+6900 \mathrm{~km} \mathrm{~s}^{-1}$ could conceivably be faint redshifted Ly $\alpha$ emission (see Section 3 and Table 1 for more detail).

(A color version of this figure is available in the online journal.)

by at least an order of magnitude if we consider only the $\mathrm{H} \alpha$ emission in the core of the line compared to Ly $\alpha$ at the same velocity (see Figure 4 and discussion in Section 4.2). As we discuss in the next section, plausible explanations are Ly $\alpha$ destruction by dust grains and the possibility that the observed $\mathrm{H} \alpha$ and $\mathrm{Ly} \alpha$ lines form in different regions.

\section{DISCUSSION}

\subsection{The FUV Continuum Emission}

Figure 3 shows the SED of Mrk 231 from $\sim 1000 \AA$ to $\sim 10,000 \AA$, where we have combined the new COS spectrum with archival FOS spectra and our 2001 Keck optical spectrum. Our COS spectrum matches up precisely with the 1996 November G160H spectrum from Gallagher et al. (2002) without rescaling; there is therefore no evidence for FUV variability. The older G190/G270H observations from 1992 November from Smith et al. (1995) are systematically lower, perhaps by as much as a factor of two, than our spectra and those of Gallagher et al.; this indicates real FUV variations and/or lost flux in the older pre-COSTAR observations. In Figure 3, we rescaled the Smith et al. G270H spectrum to join smoothly with the Gallagher et al. data. At optical wavelengths, a comparison of the Keck optical spectrum shown in the figure with our new KPNO spectrum shows no evidence for variations in the optical continuum and line emission of Mrk 231. The KPNO spectrum is not shown in Figure 3 because it does not cover as broad a wavelength range as the Keck data.

In the following, we assume, based on the results from spectroscopic and spectropolarimetric studies (e.g., Thompson et al. 1980; Schmidt \& Miller 1985; Goodrich \& Miller 1994; Smith et al. 1995, 2004; Gallagher et al. 2005), that the bulk of the optical continuum emission in Mrk 231 is produced by the AGN. The situation at shorter wavelengths is less clear. As first pointed out by Smith et al. (1995), there is a dramatic change in the SED slope around $\sim 2400 \AA$. As shown in Figure 3, the shape of the FUV-NUV spectrum, especially below $\sim 1400 \AA$, is not consistent with any reddening curve, particularly those having the $2175 \AA$ dust absorption feature (e.g., Conroy et al. 2010). The dramatic drop in flux from $\sim 4000 \AA$ to $\sim 2500 \AA$ requires $A_{V} \sim 7 \mathrm{mag}$ (or $E(B-V)=A_{V} / 3.1 \sim 2.3$ mag.; see Figure 3 ), but the continuum level shortward of $\sim 2400 \AA$ lies well above the predictions. Smith et al. (1995) attribute the bulk of the $<2400 \AA$ continuum emission to unreddened light from $\sim 10^{5}$ hot, young OB stars surrounding the active nucleus. They argue that this component dilutes the polarized light from the nucleus, resulting in the rapid decline in the degree of polarization they measure from $\sim 3200 \AA$ to $\sim 1800 \AA$.

Our COS spectrum provides crucial new constraints on this issue. Hot $\mathrm{O}$ and early $\mathrm{B}$ stars are expected to produce several stellar photospheric and wind features within the rest-frame 1100-1400 ̊: Pv $\lambda \lambda 1118,1128$, Si IV $\lambda \lambda 1122$, 1128, C III $\lambda \lambda 1176,1247, \mathrm{~N} v \lambda \lambda 1238,1243, \mathrm{O} v \lambda 1371, \mathrm{Fe} v$ $\lambda \lambda 1360-1380$, and Si IV $\lambda \lambda 1394,1403$ (e.g., Leitherer et al. 2001; Robert et al. 2003; Vázquez et al. 2004). The lack of any of these features, especially $\mathrm{C}_{\text {III }} \lambda 1176$ (EW $\lesssim 0.8 \AA$ ), indicates that hot $\mathrm{OB}$ stars cannot contribute more than $\sim 40 \%$ of the FUV continuum emission. This number is based on the C III $\lambda 1176$ equivalent width of $2 \AA$ measured by Vázquez et al. (2004) in NGC 1705-1. This estimate for the stellar contribution is a conservatively high value since we are assuming here that the weak inflection at $\sim 1225 \AA$ is entirely due to $\mathrm{C}$ III $\lambda 1176$, i.e., we neglect the possibility of kinematic substructures in the Ly $\alpha$ emission mimicking this inflection. The apparently large width $\left(\gtrsim 1000 \mathrm{~km} \mathrm{~s}^{-1} \mathrm{FWZI}\right)$ of this feature makes this identification as a stellar feature very unlikely. Indeed, our measurements of Si IV $\lambda \lambda 1394,1403$ suggest smaller contributions from hot stars.

We conclude that the AGN is the dominant source of the FUV continuum emission in our data. Both thermal and non-thermal AGN processes may in principle contribute to the observed FUV continuum emission. But, as discussed in Smith et al. (1995), synchrotron radiation from the AGN can safely be ruled out based on the fact that the polarization measurements of the strong emission lines are nearly the same as that of the nearby continuum. We thus favor the scenario where the FUV light is thermal emission from a geometrically thin, optically thick accretion disk (e.g., Davis et al. 2007 and references therein). The slowly declining FUV continuum (consistent with $F_{\lambda} \propto \lambda^{1.7}$, within the COS wavelength range) and the near-zero polarization at $\sim 1800 \AA$ (Smith et al. 1995) implies that this emission is affected only slightly by dust $\left(A_{V} \sim 0.5 \mathrm{mag}\right)$. This puts strong constraints on the dust distribution as we discuss in Section 4.3.

\subsection{Broad and Blueshifted Lya Emission}

The large blueshift of the broad Ly $\alpha$ emission in Mrk 231 $\left(\sim-3500 \mathrm{~km} \mathrm{~s}^{-1}\right)$ is unusual among AGNs. Most data on broad emission-line shifts are based on the C IV $\lambda \lambda 1548,1550$ doublet rather than Ly $\alpha$ since $\mathrm{C}$ IV is less affected by the intervening IGM (Ly $\alpha$ forest), is free of nearby line emission ( $\mathrm{N} \mathrm{v}$ blends with Ly $\alpha$ at low resolution), and is accessible from the ground starting at lower redshifts than $\operatorname{Ly} \alpha$. The mean shift of C IV among radio-quiet quasars like Mrk 231 is $-800 \mathrm{~km} \mathrm{~s}^{-1}$ (-300 for radio-loud systems; Richards et al. 2011). A shift of $-3500 \mathrm{~km} \mathrm{~s}^{-1}$ falls well beyond the distribution of C IV blueshifts measured by Richards et al. (their Figure 2, which is based on $\sim 30,000$ SDSS quasars). We come to a similar conclusion when using Ly $\alpha$ directly, although here the statistics are considerably weaker (Kramer \& Haiman 2009, based on 


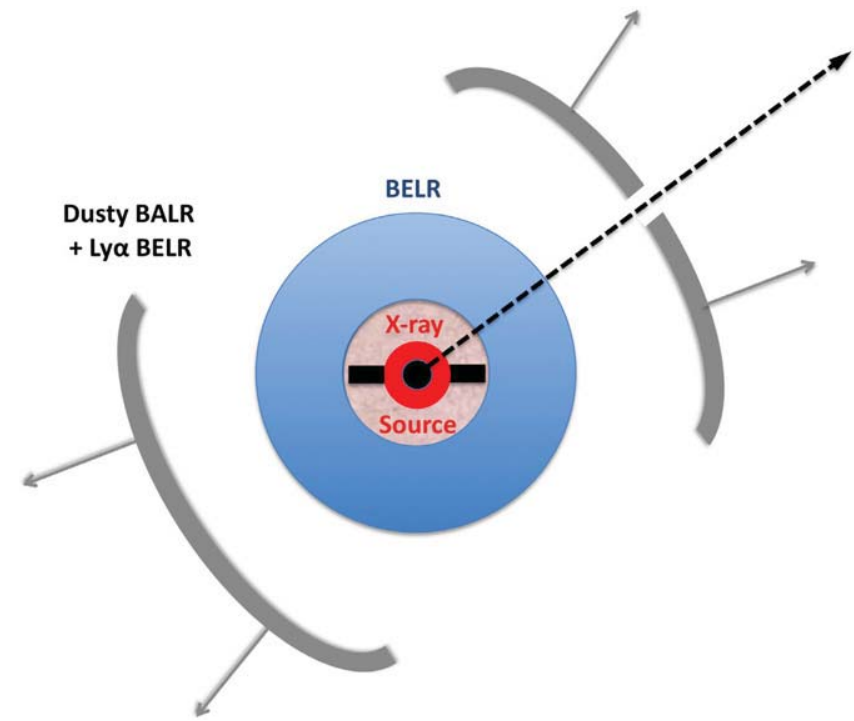

Figure 5. Simple geometric spherical model of the central pc-scale regions of Mrk 231 (not drawn to scale). The BELR (in blue) is modeled as a thick shell while the BALR (in gray) is a thin, differentially expanding dusty shell with a small hole along the line of sight to the central FUV-emitting accretion disk $(\lesssim 0.01 \mathrm{pc}$, shown in black). This small hole lets only a small fraction $(\sim 5 \%-10 \%)$ of the FUV continuum source through the shell. BELR clouds along the line of sight and hot gas (shown in pink) within the inner radius of the BELR, fully ionized by the X-ray source (shown in red), contribute to the X-ray measured Compton-thick absorbing column density. In this picture, the observed FUV continuum emission is the $\sim 5 \%-10 \%$ that goes through the hole unabsorbed. All of the BELR emission, produced on larger scale, is either completely absorbed by the shell (FUV resonance lines; e.g., Ly $\alpha$ ) or attenuated (NUV-optical lines). The weak C IV BAL measured by others suggests that the hole in the shell is actually filled with dust-free ionized material of lower density (higher ionization). Most of the Ly $\alpha$ produced in the shell itself is absorbed on the way out partly by direct absorption and partly due to photon scattering on the grains. The small fraction of the Ly $\alpha$ photons that makes it out of the shell comes from the part of the shell facing the observer, resulting in the observed large blueshift (see Section 4.3 for more detail).

(A color version of this figure is available in the online journal.)

only $\sim 80$ quasars): the median blueshift of Ly $\alpha$ in these lowredshift unobscured quasars is of the order of $-400 \mathrm{~km} \mathrm{~s}^{-1}$ and, according to their Figure 5, a shift of $-3500 \mathrm{~km} \mathrm{~s}^{-1}$ is seen in only two other (unnamed) objects. The large Ly $\alpha$ blueshift in Mrk 231 is even more unusual when we consider only FeLoBAL QSOs. In these objects, the broad absorption trough removes the blueshifted $\operatorname{Ly} \alpha$ emission, resulting in an $\operatorname{Ly} \alpha$ emission profile that is shifted to the red (e.g., Reichard et al. 2003; Trump et al. 2006; Gibson et al. 2009; Richards et al. 2011). The only objects with blueshifted emission lines similar to Ly $\alpha$ in Mrk 231 are the "PHL 1811 analog" quasars, where C IV is blueshifted by at least $-6000 \mathrm{~km} \mathrm{~s}^{-1}$ and tends to have a lower-than-average equivalent width (e.g., Leighly et al. 2007a, 2007b; Wu et al. 2011, 2012).

To gain insight into the origin of this large Ly $\alpha$ blueshift, it is instructive to compare the Ly $\alpha$ emission profile of Mrk 231 with the features detected at optical wavelengths. The results from this comparison are summarized in Figure 4, where the unscaled continuum-subtracted emission profiles of $\mathrm{Ly} \alpha$ and $\mathrm{H} \alpha$ and absorption profile of $\mathrm{Na}$ I $\mathrm{D}$ derived from our recent optical spectrum are displayed on the same velocity scale (the profiles of the other Balmer emission lines are not shown here because they are much less reliable due to lower $\mathrm{S} / \mathrm{N}$ and strong Fe II emission affecting $\mathrm{H} \beta$ and $\mathrm{H} \gamma$ ). The difference between the profiles of $\mathrm{H} \alpha$ and $\mathrm{Ly} \alpha$ is stunning. Within the uncertainties of the continuum placement, the profile of the $\mathrm{H} \alpha$ emission is symmetric, centered on the systemic velocity of Mrk 231 and with broad wings extending to at least $\pm 7500 \mathrm{~km} \mathrm{~s}^{-1}$ from systemic and a slight excess emission around $-5000 \mathrm{~km} \mathrm{~s}^{-1}$ (see also Punsly \& Lipari 2005).

The Ly $\alpha$ emission profile is a better match to the absorption profile of $\mathrm{Na}$ I D than to the emission profile of $\mathrm{H} \alpha$. While the Ly $\alpha$ emission extends to redder wavelengths than the $\mathrm{NaI}$ absorption, both of these features are highly blueshifted, with blue wings extending to at least $-8000 \mathrm{~km} \mathrm{~s}^{-1}$. This comparison suggests that the small fraction of the Ly $\alpha$ emission which is able to escape from Mrk 231 does so preferentially along the flow direction of the FeLoBAL gas (e.g., radially away from the central engine; see Hall et al. 2004 for a similar explanation of the peculiar UV Fe II spectrum of SDSS J091103.49+444630.4). This can happen naturally if the emission comes from an outflow region with a significant radial velocity gradient (e.g., Hamann et al. 1993). If the outflowing BAL systems were instead in front of the source of the $\operatorname{Ly} \alpha$ emission, then we would expect the Ly $\alpha$ emission to be suppressed, rather than preferentially transmitted, at the blueshifted velocities corresponding to the foreground Ly $\alpha$ BAL (e.g., Verhamme et al. 2006; Schaerer et al. 2011). This is seen for instance in $z \sim 3$ Lyman break galaxies, where the Ly $\alpha$ emission is redshifted by approximately twice the outflow velocity measured from interstellar absorption lines (e.g., Shapley et al. 2003). Moreover, the BALR would have to cover the BELR without covering the FUV continuum source-otherwise, one would have to tune the BAL optical depths to get transmitted fluxes nearly equal to the continuum at adjacent wavelengths - but we already know from the optical spectra (e.g., Rupke et al. 2002) that the BAL gas covers at least $80 \%-90 \%$ of the optical continuum source (it is a lower limit because light from an old stellar population may also contribute to the residual light in the Na I D trough; Smith et al. 1995). To avoid these difficulties, we favor a scenario where most, if not all, of the observed Ly $\alpha$ emission is produced in the outflowing material itself and is not part of the standard BELR; this scenario is discussed next.

\subsection{Physical Model}

The data on Mrk 231 are complex with different wavelengths and features each providing different clues on the geometry of the central engine. Figures 5 and 6 show two simple pictures that attempt to explain the main features of these data, particularly the peculiar Ly $\alpha$ emission, the slowly declining and nearly unpolarized FUV continuum, and the absence of broad absorption features in the FUV despite the presence of a strong optical-NUV FeLoBAL outflow. These simple models are no doubt simplifications of the reality and may not explain all of the details in the observations. In both pictures, the outflowing BALR acts as a partial screen for the continuum emission and as the source of the observed Ly $\alpha$ emission. We discuss these pictures separately, although the possibility that Mrk 231 is a hybrid of both cannot be ruled out.

Figure 5 (hereafter referred to as the "spherical geometry" or "spherical scenario") is an attempt to explain the data using the simplest geometry possible: the standard BELR is a thick spherical shell surrounded by a thin dusty shell representing the outflowing BALR. The source of the FUV continuum is an accretion disk on the scale of $\sim$ few $\times 10^{15} \mathrm{~cm}(\lesssim 0.01 \mathrm{pc})$ with arbitrary orientation. If this geometry is to explain BAL QSOs in general, particularly their low frequency of occurrence among QSOs, then the BALR shell must subtend only a small fraction of $4 \pi$ str or be present for only a fraction of the QSO lifetime (e.g., 


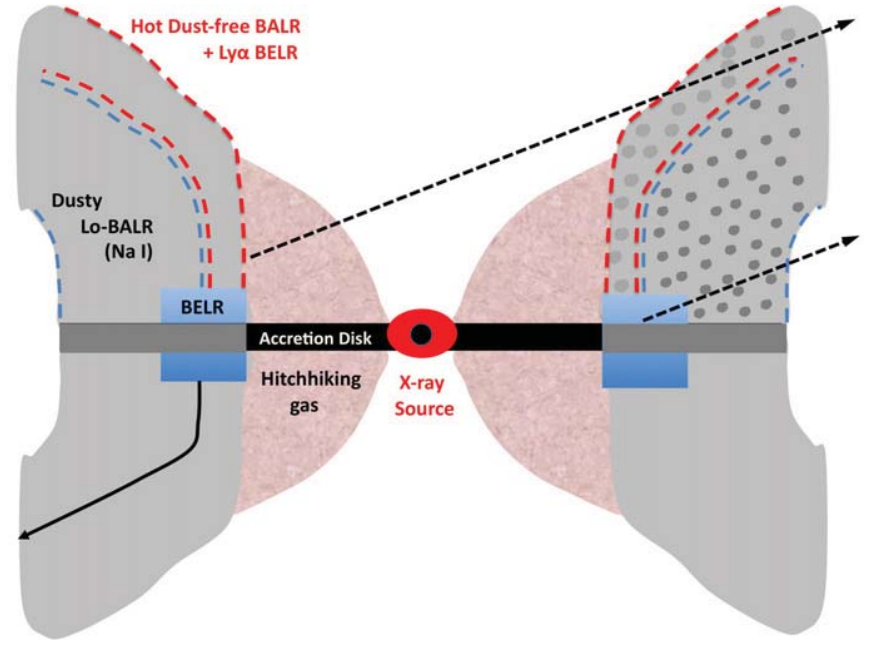

Figure 6. Physically motivated geometric disk model of the central pc-scale regions of Mrk 231 (not drawn to scale). A strong clumpy outflow (shown in gray, with a typical flow line shown in solid black) is present, lifted off the accretion disk (in dark gray), and radiatively accelerated by the UV radiation field from the UV-emitting accretion disk (in black). Gas too close to the central X-ray source (shown in pink and dubbed the "hitchhiking gas" by Murray et al. 1995 ) is too ionized and thus too transparent to UV radiation to take part in this wind; it does however contribute significantly to the X-ray absorbing column density. The portion of the outflow which is closest to the UV and X-ray source is hot and free of dust; it is responsible for the weak CIV BAL feature and some of the broad line emission, particularly the blueshifted Ly $\alpha$. The densest and most radiatively shielded environment at the base of the flow is neutral and dusty; it is responsible for the strong Na I BAL seen in the optical. The boundaries between the various regions labeled in this figure are gradual and probably much more irregular than shown here. Two lines of sight are shown for illustration. The top one goes through the hot dust-free $\left(A_{V} \sim 0 \mathrm{mag}\right)$ BALR, where all of the observed Ly $\alpha$ emission, most of the C IV emission, and some Balmer line emission are produced. This line of sight is not aligned with the gas flow in the far-side dust-free BALR, so very little Ly $\alpha$ emission from this region makes it out in our direction. The FUV continuum detected along this line of sight is seen through the hot dust-free BAL, producing the faint C IV BAL. The lower line of sight goes through the patchy dusty BALR $\left(A_{V} \sim 7 \mathrm{mag}\right)$ where most of the optical continuum passes through the Na I BALR clouds (hence it is reddened and partly polarized). The small fraction of the optical and UV continuum that does not pass through the Na I BALR clouds is unpolarized; this accounts for only a small fraction of observed optical continuum, but perhaps a significant fraction of the observed FUV flux (see Section 4.3 for more detail).

(A color version of this figure is available in the online journal.)

merger-driven evolutionary scenario of Sanders et al. 1988; see also Veilleux et al. 2009; note that the covering factor may be much larger among dust-reddened quasars like Mrk 231 and those selected via a FIRST-2MASS cross-correlation; e.g., Urrutia et al. 2009; Glikman et al. 2012). In Mrk 231, this shell has a small dust-free "hole" along our line of sight to the FUV source that lets $5 \%-10 \%$ of the FUV continuum through the shell without being attenuated, but a negligible fraction of the BELR emission goes through this hole since the projected area of the BELR is much larger than that of the UV source. In this picture, all of the Ly $\alpha$ emission detected in Mrk 231 is produced in the dusty BALR shell. We return to this point below.

Figure 6 (hereafter called the "disk geometry" or "disk scenario") is more physically motivated but also more complicated. It is inspired by Gallagher et al. (2002; their Figure 12) and models where the BELR is comprised of two connected components, the extended accretion disk atmosphere and a radiation pressure-driven wind farther out (e.g., Murray et al. 1995; Murray \& Chiang 1995; Proga et al. 2000, 2008; Elvis 2000; Chelouche \& Netzer 2003; Proga \& Kallman 2004; Proga 2007; Richards et al. 2011; Kruczek et al. 2011; Richards 2012). In these models, the relative emission line intensities from these two components depend on the physical conditions in the outflow and therefore, perhaps, the ionizing spectral energy distribution (SED), as seen by the atmosphere of the accretion disk. The BELR and BALR are comprised of clouds/filaments of various sizes, optical depths, dust content, and ionization structures (e.g., dusty cold core + hot extended halo); only broad trends in some key physical properties are shown in Figure 6. The boundaries between the BELR and BALR are gradual and probably much more irregular than shown in this figure. As illustrated in Figure 6 and described below, the optical-UV spectra seem to favor a line of sight to Mrk 231 that skirts the upper edge of the dusty wind.

As discussed in Section 4.2, the peculiar blueshifted Ly $\alpha$ emission in Mrk 231 appears to be direct evidence for line emission associated with some of the nuclear outflowing material. The Ly $\alpha$ emission from a large covering factor outflowing optically thick (in the Lyman continuum) shell is predicted to be an order of magnitude or more larger than the flux we observe. Thus, only a few percent of the line photons need to escape on the blue side of the line to agree with the observations. This suggests two different ways that are conveyed by the two scenarios shown in Figures 5 and 6. The first, illustrated in Figure 5, is a large covering factor dusty shell containing very low ionization gas (low ionization parameter) and undergoing large differential expansion. The dust in this shell absorbs most of the locally emitted Ly $\alpha$ photons. The only line photons that reach the observer are the ones escaping from the part of the shell moving in this direction. The second scenario, illustrated in Figure 6, is a dust-free shell with a small covering factor and/or a small Lyman continuum optical depth. In this case, the intrinsically produced Ly $\alpha$ line is very weak and most of it escapes the gas.

A key issue in Figures 5 and 6 is the location of the dust. As mentioned earlier, the spectropolarimetric study of Smith et al. (1995) has reported near-zero polarization at $\sim 1800 \AA$, which indicates that the FUV continuum emission is not significantly affected by the dust that is causing the optical continuum polarization. The dust distribution must therefore have holes or be patchy, covering only a fraction of the FUV continuum source. Given the small size of this source, i.e., the inner accretion disk (nominally $\lesssim 0.01 \mathrm{pc}$ across), the dust patches and "holes" between the patches must themselves be very small and, therefore, they are likely to reside near the UV source rather than in the extended host galaxy (this is also the conclusions of the spectropolarimetric studies using different arguments).

At optical wavelengths, we know that a very low ionization BAL outflow (with substantial Na I, whose ionization potential is only $5.1 \mathrm{eV}$ ) covers at least $80 \%-90 \%$ of the nuclear optical continuum source along our lines of sight (e.g., Rupke et al. 2002). A few simple experiments with the photoionization code CLOUDY indicate that dust is not only likely present in this BALR, it seems to be required to shield $\mathrm{Na}$ I from the intense UV ( $\gtrsim 5 \mathrm{eV}$ or $\lesssim 2400 \AA$ ) radiation field of the AGN that would otherwise photoionize it to Na II. So, in both Figures 5 and 6, the dusty BALR may be the patchy dust absorber causing the optical continuum polarization (see also Rudy et al. 1985 and Goodrich \& Miller 1994 for independent supportive arguments). If the FUV continuum source is also not completely covered by the dusty BALR, then the net observational result could be just what we observe in Mrk 231: a red partly polarized optical continuum where at least $80 \%-90 \%$ of the flux is transmitted through the dusty BALR plus a blue nearly unpolarized FUV continuum 
spectrum dominated by the small fraction of the FUV source not covered substantially by the dusty BALR. This explanation for the blue FUV spectrum is strongly supported by the absence of neutral and low-ionization BALs in the FUV, because the observed FUV flux is not transmitted through this part of the BALR.

Both suggested scenarios are not without difficulties. In the spherical geometry of Figure 5, one concern is that a single hole that transmits only $\sim 5 \%-10 \%$ of the FUV continuum emission may not be able to explain the considerable amount of unpolarized residual light in the troughs of the $\mathrm{Na}$ I $\mathrm{D}$ absorption (10\%-20\% of the continuum at $\sim 6000 \AA$, depending on aperture size; e.g., Rupke et al. 2002). The optical continuum source in a thin accretion disk is predicted to be about an order of magnitude larger than the source of the FUV continuum (size $\sim \lambda^{4 / 3}$ ), so a significantly smaller fraction of the optical continuum should make it out through the single hole. A patchy absorber could explain this result if the gaps or holes between patches are typically smaller than any continuum source, but this patchy absorber would also let $\sim 5 \%-10 \%$ of the BELR emission through the shell without being attenuated, which seems inconsistent with the small $\mathrm{Ly} \alpha / \mathrm{H} \alpha$ emission ratio. However, there are a number of issues with these arguments. First, as mentioned earlier, light from an old stellar population may contribute significantly to the residual flux in the $\mathrm{NaI}$ BAL troughs. This would alleviate the problem by reducing the fraction of the optical continuum emission from the accretion disk that gets through the BALR. The fact that the fraction of residual light in the $\mathrm{Na}$ I troughs is lower when using smaller apertures is consistent with this idea. The slightly positive slope $\left(\sim \lambda^{1.7}\right)$ of the FUV continuum, attributed to reddening with $A_{V} \sim 0.5 \mathrm{mag}$, predicts a larger contribution of (nearly) unpolarized light at optical wavelengths than in the FUV, which would help explain the optical data. Finally, recent microlensing determinations of continuum source sizes in quasars (e.g., Blackburne et al. 2011) suggest that the source of continuum emission is larger by nearly an order of magnitude than the predictions from the standard thin disk models and nearly independent of wavelengths $\left(\sim \lambda^{0.2}\right.$ instead of $\left.\lambda^{4 / 3}\right)$. The simple single-hole model may therefore be able to explain the lowpolarization FUV continuum, the faint residual light in the $\mathrm{Na}$ I troughs, and the small $\mathrm{Ly} \alpha / \mathrm{H} \alpha$ emission ratio all at once.

A second, perhaps more serious, concern with the spherical scenario, where a single hole transmits nearly all of the observed FUV continuum emission, is the apparent lack of significant UV continuum variability in Mrk 231 over a span of at least 15 years (based on the 1996 FOS and 2011 COS data). The hole will change shape and eventually move across our line of sight to the small FUV continuum source, resulting in large changes in the amount of FUV emission reaching the observer. But the timescale for these changes depends on several factors (e.g., radius of the BALR shell, size of the hole, and density contrast between the shell and the hole), which can only be explored with a proper theoretical treatment that is beyond the scope of the present paper. A third concern with Figure 5 has to do with the need for the BALR to redden the NUV-optical continuum by $A_{V} \sim 7 \mathrm{mag}$ and to produce detectable BAL features in not only $\mathrm{Na} \mathrm{I}$ and $\mathrm{Ca}$ II $\mathrm{H}$ and $\mathrm{K}$ but also He I* $\lambda 3889$ (which arises from a metastable excited state populated by recombination from He II; Leighly et al. 2011). This suggests the need for neutral and moderately ionized columns $\log N_{\mathrm{H}} \gtrsim 10^{22} \mathrm{~cm}^{-2}$. Again, it is hard to tell without a proper theoretical treatment if enough Ly $\alpha$ photons are able to escape from this thick shell.
In the disk geometry of Figure 6, the dusty BALR is in the densest and most radiatively shielded environment at the base of the flow. This inner BALR extinguishes our view of the BELR and, therefore, helps to suppress the broad emission lines in the FUV. Dust emerging from the disk might further suppress emission from the BELR, especially for a strong resonance line like Ly $\alpha$. The weak blueshifted Ly $\alpha$ emission profile that we do observe could be exempt from this BALR extinction if it forms a little farther out in the BALR, above the inner dusty region (see Figure 6). In this picture, the observed Ly $\alpha$ emission comes from the near-side portion of hot dust-free BALR, while the Balmer lines arise primarily from the standard BELR seen through the dusty BALR, in addition to contributions from the dust-free BALR and possibly also the dusty BALR (hence explaining the large $\mathrm{H} \alpha / \mathrm{Ly} \alpha$ emission-line ratio and a more symmetric $\mathrm{H} \alpha$ profile). The observed lack of UV continuum variability in Mrk 231 is a weaker concern in this picture because part of the UV continuum emission reaches the observer through the dust-free BALR, while the rest comes through the holes (rather than a single hole) of the patchy $\mathrm{Na}$ I BALR. A potentially more serious problem is the implied orientation of the disk. In the idealized disk geometry of Figure 6, the absence of redshifted Ly $\alpha$ emission in Mrk 231 (except perhaps for the faint emission feature at $\sim 1296 \AA$, see Section 3, Table 1) implies a rather edge-on view of the disk with $i \gtrsim 45^{\circ}$ so that the far-side portion of the dust-free BALR is largely obscured by the dusty BALR on the near-side. On the other hand, the results from mmwave/infrared studies on larger few $100 \mathrm{pc}$ scales indicate that Mrk 231 has a disk viewed rather face on $\left(i=10^{\circ}-20^{\circ}\right.$; e.g., Downes \& Solomon 1998; Davies et al. 2004). These results can be reconciled if this $100 \mathrm{pc}$ scale disk is tilted significantly with respect to the accretion disk. The presence of a warp has in fact been inferred in the inner $\sim 200$ pc by Davies et al. (2004) and results on smaller scales (e.g., Carilli et al. 1998; Ulvestad et al. 1999; Klockner et al. 2003; $i=56^{\circ}$ ) seem to suggest a more edge-on orientation that provides support for this picture. In reality, the boundaries between the dusty and dustfree BALRs are probably much more irregular than shown in that figure, so the constraint on the orientation may be relaxed. Moreover, the "puffiness" of the dusty BALR, which sets the covering factor of the FeLoBAL outflow, is poorly constrained but likely high in Mrk 231. The derived fraction of LoBALs among quasars depends on the methods of selection, ranging from only a few percents among UV- or optically selected quasars to more than $50 \%$ in the FIRST-2MASS red quasars, more akin to Mrk 231 (e.g., Urrutia et al. 2009; Glikman et al. 2012). Also as mentioned above, the line emission from the BALR could be mostly in the forward (radial) direction if there are sufficient velocity gradients to create this avenue for escape. Since our line of sight is not aligned with the gas flow in the farside dust-free BALR, it may be that very little emission from this region makes it out in our direction. Finally, it is also possible that the line emission from the far-side BELR and dust-free BALR is attenuated not only by the foreground dusty BALR but also by other intervening gas, including the hitchhiking gas which is nearly Compton thick at least along some sight lines (Braito et al. 2004; Morabito et al. 2011).

The weak but non-zero C IV BAL detected by Gallagher et al. (2002) is expected in both scenarios. In the disk geometry, it happens naturally along the line of sight that goes through the hot dust-free BALR. In the spherical geometry of Figure 5, this feature may be explained if the dust-free hole is filled with ionized gas of lower density (higher ionization) that 
has presumably leaked out of the shell. The lack of obvious blueshifted Si IV $\lambda \lambda 1394,1403$ BAL in the presence of weak $\mathrm{C}$ IV BAL is also not surprising since $\mathrm{Si}$ IV has a lower ionization potential and lower elemental abundance (in the Sun) than C IV, and it is not uncommon to see C IV BALs accompanied by weak or absent Si IV in HiBALs, i.e., the more highly ionized absorbers (e.g., Trump et al. 2006; Gibson et al. 2009; Allen et al. 2011). The lack of $\mathrm{N} v \lambda \lambda 1238,1243$ with $\mathrm{C}$ IV is perhaps harder to explain in this context since the ionization potential of $\mathrm{N}^{+4}$ is $77 \mathrm{eV}$ and the $\mathrm{N} v$ transitions are generally present in HiBALs, although noticeably weaker than C IV. As discussed in Section 3 (Table 1), the emission feature at $1296 \AA$ may conceivably be residual $\mathrm{N} \mathrm{v}$ (+ redshifted Ly $\alpha$ ) emission affected by broad $\mathrm{N} \mathrm{v}$ absorption, but this scenario is a bit contrived. Perhaps a more plausible explanation is that the C IV HiBAL detected in the FOS spectra obtained in 1996 has since disappeared without affecting the Na I BAL. This sort of HiBAL variability would not be unusual (e.g., Gibson et al. 2008; the $0.5-2 \mathrm{keV}$ and 2-10 keV fluxes of Mrk 231 have increased by more than $50 \%$ from 2001 to 2011; Piconcelli et al. 2013). New COS spectroscopy covering Ly $\alpha$ and C IV $\lambda \lambda 1548,1550$ simultaneously would easily settle the issue.

\section{SUMMARY AND FINAL REMARKS}

We have recently obtained a high $\mathrm{S} / \mathrm{N}$ FUV spectrum of Mrk 231 covering $\sim 1100-1450 \AA$ as part of our spectroscopic survey of nearby QSOs with HST-COS. The spectrum presents faint, broad, and highly blueshifted Ly $\alpha$ emission. The FUV continuum emission is slowly declining at shorter wavelengths and shows no sign of stellar photospheric or wind features and no obvious BAL troughs like those seen in the NUV-optical spectrum of this FeLoBAL QSO. These data are best explained if the FUV continuum emission is produced by the accretion disk of the AGN and the observed Ly $\alpha$ emission is produced in the outflowing BAL cloud systems. The BAL clouds act as a patchy screen for the FUV continuum emission and as a polar scattering agent to reproduce the distinct optical polarization signatures in this object. Many of these results may be explained using a simple spherical geometry or a physically motivated but more complex disk geometry (Figures 5 and 6). Both of these scenarios are not without problems, however. A proper theoretical treatment beyond the scope of the present paper will be needed to decide which scenario, if any, is favored over the other.

One may wonder why other FeLoBAL QSOs do not show the same complex UV-optical spectrum as Mrk 231. The detection of the Na I BAL in Mrk 231 is an important clue since it requires dust. In the disk scenario, Mrk 231 may be an extreme case of the classic BAL outflow/dusty torus model of Konigl \& Kartje (1994) with larger column density, higher mass-loss rate, and hence more shielding, than usual. This extra shielding allows dust to survive at smaller radii. This facilitates the appearance of dust (and $\mathrm{Na} \mathrm{I}$ ) in the BALR, as well as the formation of a dusty toroidal wind farther out. This extreme outflow also leads to more broad line emission from a wind component of the BELR (e.g., Reichard et al. 2003; Richards et al. 2011). The amount of covering by this dusty BALR, which is inferred from the depths of the Ca II and Na I troughs (Rupke et al. 2002; Smith et al. 1995), is consistent with the amount of line-ofsight covering needed to explain the residual FUV continuum, roughly $80 \%-90 \%$.

Note finally that in both the spherical and disk geometries of Figures 5 and 6 , the BALR is located within the nuclear
$<1-10$ pc scale region rather than on galactic kpc scales. The location of the BALR is an important issue since the outflow energetics scale with the distance of the BALR from the central engine and thus have a direct bearing on the role of quasar feedback in the evolution of galaxies. Recently, FaucherGiguère et al. (2012) modeled FeLoBAL outflows as ISM clouds (on $\sim \mathrm{kpc}$ scales) that are shocked, shredded, and accelerated by a hot (normal HiBAL) quasar wind. This is inspired by the observational work of, e.g., Moe et al. (2009), Bautista et al. (2010), and Dunn et al. (2010) indicating that some of those flows, or some (narrow line) components of them, do exist at $>\mathrm{kpc}$ distances. This model likely does not apply to Mrk 231 for the following reasons: (1) the Na I BALs of Mrk 231 are not spatially resolved on sub-kpc scale (Rupke et al. 2002; Rupke \& Veilleux 2011, 2012). (2) The Na I BALs varied, at least in the highest velocity components. This puts Mrk 231 in line with several other BAL variability studies and, in particular, with the work by Hall et al. (2011) on a variable FeLoBAL, that favors pc-scale origins for the BALs. (3) The detached high-velocity Na I troughs in Mrk 231, which imply a lack of nuclear absorbers with velocities between $\sim-3500$ and $0 \mathrm{~km}$ $\mathrm{s}^{-1}$, are difficult to explain in the models of Faucher-Giguère et al. (2012), where the cold gas is accelerated by the quasar blast wave starting at the near-zero velocity of the ambient ISM. (4) Several spectropolarimetric studies of Mrk 231 have suggested that the FeLoBAL clouds are causing the optical continuum polarization, which is due to dust scattering in the polar illumination cones of the circumnuclear region, roughly along the pc-scale jet axis of Mrk 231. This result would be hard to explain if the FeLoBAL clouds were distributed on a galactic scale, where the influence of the central engine is much more isotropic (Rupke \& Veilleux 2011, 2012). While these arguments favor a nuclear origin for the FeLoBAL system in Mrk 231, none of them rule out the possibility that this type of shocked and accelerated ISM model may apply to the spatially resolved galactic-scale outflow in this object (Rupke \& Veilleux 2011, 2012; Faucher-Giguère \& Quataert 2012).

Support for this work was provided to S.V., M.T., and T.M.T. by NASA through contracts HST GO-1256901A and GO-1256901B. S.V. also acknowledges support from a Senior NPP Award held at the NASA Goddard Space Flight Center, where most of this paper was written, and from the Humboldt Foundation to provide funds for a long-term visit at MPE in 2012. F.H. acknowledges support from the National Science Foundation through grant AST-0908910. We thank the referee, Patrick Hall, for a thorough report and thoughtful suggestions which improved the paper. S.V., F.H., and D.L. also thank A. Laor and H. Netzer for organizing an excellent AGN meeting in Haifa, where many of the ideas presented here germinated. This work has made use of NASA's Astrophysics Data System Abstract Service and the NASA/IPAC Extragalactic Database (NED), which is operated by the Jet Propulsion Laboratory, California Institute of Technology, under contract with the National Aeronautics and Space Administration.

\section{REFERENCES}

Aalto, S., Garcia-Burillo, S., Muller, S., et al. 2012, A\&A, 537, 44

Allen, J. T., Hewett, P. C., Maddox, N., Richards, G. T., \& Belokurov, V. 2011, MNRAS, 410, 860

Bautista, M., Dunn, J. P., Arav, N., et al. 2010, ApJ, 713, 25

Blackburne, J. A., Pooley, D., Rappaport, S., \& Schechter, P. L. 2011, ApJ, 729,34 
Boksenberg, A., Carswell, R. F., Allen, D. A., et al. 1977, MNRAS, 178, 451 Boroson, T. A., Meyers, K. A., Morris, S. L., \& Persson, S. E. 1991, ApJL, 370, 19

Braito, V., Della Ceca, R., Piconcelli, E., et al. 2004, A\&A, 420, 79

Carilli, C. L., Wrobel, J. M., \& Ulvestad, J. S. 1998, AJ, 115, 928

Chelouche, D., \& Netzer, H. 2003, MNRAS, 344, 233

Cicone, C., Feruglio, C., Maiolino, R., et al. 2012, A\&A, 543, 99

Conroy, C., Schiminovich, D., \& Blanton, M. R. 2010, ApJ, 718, 184

Danforth, C. W., Keeney, B. A., Stocke, J. T., Shull, J. M., \& Yao, Y. 2010, ApJ, 720, 976

Davies, R. I., Tacconi, L. J., \& Genzel, R. 2004, ApJ, 613, 781

Davis, S. W., Woo, J.-H., \& Blaes, O. M. 2007, ApJ, 668, 682

Di Matteo, T., Springel, V., \& Hernquist, L. 2005, Natur, 433, 604

Downes, D., \& Solomon, P. M. 1998, ApJ, 507, 615

Dunn, J. P., Bautista, M., Arav, N., et al. 2010, ApJ, 709, 611

Elvis, M. 2000, ApJ, 545, 63

Faucher-Giguère, C.-A., \& Quataert, E. 2012, MNRAS, 425, 605

Faucher-Giguère, C.-A., Quataert, E., \& Murray, N. 2012, MNRAS, 420, 1347

Feruglio, C., Maiolino, R., Piconcelli, E., et al. 2010, A\&A, 518, L155

Fischer, J., Sturm, E., González-Alfonso, E., et al. 2010, A\&A, 518, L41

Forster, K., Rich, R. M., \& McCarthy, J. K. 1995, ApJ, 450, 74

Gallagher, S. C., Brandt, W. N., Chartas, G., Garmire, G. P., \& Sambruna, R. M. 2002, ApJ, 569, 655

Gallagher, S. C., Schmidt, G. D., Smith, P. S., et al. 2005, ApJ, 633, 71

Ghavamian, P., Froning, C., Osterman, S., et al. 2010, in Proc. 2010 Space Telescope Science Institute Workshop: Hubble after SM4, ed. S. Deustua \& C. Oliveira, 46

Gibson, R. R., Brandt, W. N., Schneider, D. P., \& Gallagher, S. C. 2008, ApJ, 675,985

Gibson, R. R., Jiang, L., Brandt, W. N., et al. 2009, ApJ, 692, 758

Glikman, E., Urrutia, T., Lacy, M., et al. 2012, ApJ, 757, 51

Goodrich, R. W., \& Miller, J. S. 1994, ApJ, 434, 82

Green, J. C., Froning, C. S., Osterman, S., et al. 2012, ApJ, 744, 60

Hall, P. B., Anderson, S. F., Strauss, M. A., et al. 2002, ApJS, 141, 267

Hall, P. B., Knapp, G. R., Richards, G. T., et al. 2004, in Multiwavelength AGN

Surveys, ed. R. Mujica \& R. Maiolino (Singapore: World Scientific), 247

Hall, P. B., Anosov, K., White, R. L., et al. 2011, MNRAS, 411, 2653

Hamann, F., Korista, K. T., \& Morris, S. L. 1993, ApJ, 415, 541

Hamilton, D., \& Keel, W. C. 1987, ApJ, 321, 211

Hutchings, J. B., \& Neff, S. G. 1987, AJ, 92, 14

Keeney, B. A., Danforth, C. W., Stocke, J. T., France, K., \& Green, J. C. 2012, PASP, 124, 830

Klockner, H.-R., Baan, W. A., \& Garrett, M. A. 2003, Natur, 421, 821

Kollatschny, W., Dietrich, M., \& Hagen, H. 1992, A\&A, 264, L5

Konigl, A., \& Kartje, J. F. 1994, ApJ, 434, 446

Kramer, R. H., \& Haiman, Z. 2009, MNRAS, 400, 1493

Kruczek, N. E., Richards, G. T., Gallagher, S. C., et al. 2011, AJ, 142, 130

Laor, A., Jannuzi, B. T., Green, R. F., \& Boroson, T. A. 1997, ApJ, 489, 656

Leighly, K. M., Dietrich, M., \& Barber, S. 2011, ApJ, 728, 94

Leighly, K. M., Halpern, J. P., Jenkins, E. B., et al. 2007a, ApJ, 663, 103

Leighly, K., Halpern, J. P., Jenkins, E. B., \& Casebeer, D. 2007b, ApJS, 173, 1

Leitherer, C., Leão, J. R. S., Heckman, T. M., et al. 2001, ApJ, 550, 724

Lipari, S., Colina, L., \& Macchetto, F. 1994, ApJ, 427, 174
Lipari, S., Sanchez, S. F., Bergmann, M., et al. 2009, MNRAS, 392, 1295

Moe, M., Arav, N., Bautista, M. A., \& Korista, K. T. 2009, ApJ, 706, 525

Morabito, L. K., Dai, X., Leighly, K. M., Sivakoff, G. R., \& Shankar, F. 2011, ApJ, 737, 46

Morton, D. C. 2003, ApJS, 149, 205

Murray, N., \& Chiang, J. 1995, ApJL, 454, 105

Murray, N., Quataert, E., \& Thompson, T. A. 2005, ApJ, 618, 569

Murray, N., Chiang, J., Grossman, S. A., \& Voit, G. M. 1995, ApJ, 451, 498

Netzer, H., Brotherton, M. S., Wills, B. J., et al. 1995, ApJ, 448, 27

Piconcelli, E., Miniutti, G., Ranalli, P., et al. 2013, MNRAS, 428, 1185

Proga, D. 2007, ApJ, 661, 693

Proga, D., \& Kallman, 2004, ApJ, 616, 688

Proga, D., Ostriker, J. P., \& Kurosawa, R. 2008, ApJ, 676, 101

Proga, D., Stone, J. M., \& Kallman, T. R. 2000, ApJ, 543, 686

Punsly, B., \& Lipari, S. 2005, ApJL, 623, 101

Reichard, T. A., Richards, G. T., Hall, P. B., et al. 2003, AJ, 126, 2594

Richards, G. T. 2012, in ASP Conf. Ser. 460, AGN Winds in Charleston, ed. G. Chartas, F. Hamann, \& K. M. Leighly (San Francisco, CA: ASP), 67 Richards, G. T., Kruczek, N. E., Gallagher, S. C., et al. 2011, ApJ, 141, 167

Robert, C., Pellerin, A., Aloisi, A., et al. 2003, ApJS, 144, 21

Rudy, R. J., Foltz, C. B., \& Stocke, J. T. 1985, ApJ, 288, 531

Rupke, D. S. N., \& Veilleux, S. 2011, ApJL, 729, 27

Rupke, D. S. N., \& Veilleux, S. 2012, ApJ, submitted

Rupke, D. S. N., Veilleux, S., \& Sanders, D. B. 2002, ApJ, 570, 588

Rupke, D. S. N., Veilleux, S., \& Sanders, D. B. 2005, ApJ, 632, 751

Sanders, D. B., \& Mirabel, I. F. 1996, ARA\&A, 34, 749

Sanders, D. B., Soifer, B. T., Elias, J. H., et al. 1988, ApJ, 325, 74

Schaerer, D., Hayes, M., Verhamme, A., \& Teyssier, R. 2011, A\&A, 531, 12

Schmidt, G. D., \& Miller, J. S. 1985, ApJ, 290, 517

Shapley, A., Steidel, C. C., Pettini, M., et al. 2003, ApJ, 588, 65

Smith, J. E., Robinson, A., Alexander, D. M., et al. 2004, MNRAS, 350, 140

Smith, P. S., Schmidt, G. D., Allen, R. G., \& Angel, J. R. P. 1995, ApJ, 444, 146

Sturm, E., González-Alfonso, E., Veilleux, S., et al. 2011, ApJL, 733, 16

Surace, J., Sanders, D. B., Vacca, W. D., Veilleux, S., \& Mazzarella, J. M. 1998, ApJ, 492, 116

Thompson, I., Stockman, H. S., Angel, J. R. P., \& Beaver, E. A. 1980, MNRAS, 192,53

Trump, J. R., Hall, P. B., Reichard, T. A., et al. 2006, ApJS, 165, 1

Ulvestad, J., Wrobel, J. M., \& Carilli, C. L. 1999, ApJ, 516, 127

Urrutia, T., Becker, R. H., White, R. L., et al. 2009, ApJ, 698, 1095

van den Berk, D. E., Richards, G. T., Bauer, A., et al. 2001, AJ, 122, 549

Vázquez, G., Leitherer, C., Heckman, T. M., et al. 2004, ApJ, 600, 162

Veilleux, S. 2012, JPhCS, 372, 012001

Veilleux, S., Cecil, G., \& Bland-Hawthorn, J. 2005, ARA\&A, 43, 769

Veilleux, S., Kim, D.-C., \& Sanders, D. B. 2002, ApJS, 143, 315

Veilleux, S., Kim, D.-C., Peng, C. Y., et al. 2006, ApJ, 643, 707

Veilleux, S., Rupke, D. S. N., Kim, D.-C, et al. 2009, ApJS, 182, 628

Verhamme, A., Schaerer, D., \& Maselli, A. 2006, A\&A, 460, 397

Wu, J., Brandt, W. N., Anderson, S. F., et al. 2012, ApJ, 747, 10

Wu, J., Brandt, W. N., Hall, P. B., et al. 2011, ApJ, 736, 28

Zheng, W., Kriss, G. A., Telfer, R. C., Grimes, J. P., \& Davidsen, A. F. 1997, ApJ, 475,469 